\title{
Study on the Pharmacological Mechanism of Phthalazinone Derivatives as Potential Drugs for Alzheimer's Disease Treatment by Network Pharmacology Analysis and Molecular Docking
}

\section{Huimin Ye}

Guangdong Pharmaceutical University https://orcid.org/0000-0002-7023-1707

Zhong Li ( $\sim$ lizhongyxy@126.com )

Guangdong Pharmaceutical University

\section{Research}

Keywords: network pharmacology, molecular docking, phthalazinone derivatives, $A D$

Posted Date: November 3rd, 2020

DOI: https://doi.org/10.21203/rs.3.rs-97683/v1

License: (c) (1) This work is licensed under a Creative Commons Attribution 4.0 International License. Read Full License 


\section{Abstract}

Objective: To screen the bioactivity of phthalazinone derivatives for AD treatment and investigate the potential pharmacological mechanism, the network pharmacology analysis and molecular docking were adopted in this study.

Methods: Those phthalazinone derivatives with certain structures and physical properties were screened out by Pubchem database in this study. Besides, to explore the potential activity of these phthalazinone derivatives as drugs for $A D$ treatment, network pharmacology study was employed, including targets prediction, gene enrichment analysis and network analysis. Network analysis of AD approved drugs and molecular docking studies were also adopted to further investigate drug-likeness of phthalazinone derivatives for $A D$ treatment.

Results: Five compounds and 57 common targets were recognized and adopted to the construction of compounds-targets network. 15 approved drugs with clear indication for AD were figured out, with 57 associated targets that originated from Homo sapiens. The KEGG enrichment analysis showed that phthalazinone derivatives and approved drugs shared the same essential pathway (neuroactive ligandreceptor interaction) and other important pathways with associated targets. The result of molecular docking indicated that these phthalazinone derivatives could interact with essential targets stably.

Conclusion: In silico analysis suggested that these derivatives are probable to be effective for the treatment of AD by interacting with the essential targets and initiating ATP binding, signal transduction, finally regulating neuroactive ligand-receptor interaction pathway $\llbracket$ calcium signaling pathway, and so on.

\section{Introduction}

Alzheimer's Disease (AD) characterized by memory impairment, aphasia, personality changes and other recognition dementia symptoms, is a kind of neurodegenerative disease with a high incidence among the aged ${ }^{[1,2]}$. Several scientists shed light on the etiology of $A D$, investigating the pharmacological mechanism of potential drugs for $A D$ treatment. So far, many factors have been found contributed to the morbidity of $A D$, concluding the insufficiency of acetylcholine (Ach), aggregation of $\beta$-amyloid ( $A \beta$ ) peptides, phosphorylation of Tau Protein and so on ${ }^{[3-8]}$. However, no pharmacological mechanism was found to be well-recognized. Donepezil and Memantine Hydrochloride, respectively representing acetylcholine esterase inhibitors(AchEI) and N-methyl-D-aspartic acid receptor (NMDAR) antagonists, are commonly utilized for the treatment of $A D$, but they also have some side effects, such as dizziness, nausea, and vomiting ${ }^{[9]}$. Many potential drugs for $A D$ treatment were withdrawn in the period of clinical trials because of severe side effects ${ }^{[10]}$. It is still of great necessity to develop new drugs for the treatment of $A D$.

According to the available literatures, the phosphodiesterase 4 (PDE4) subtype could be a potential target for the treatment of $A D$, mainly by inhibiting the aggression of $A \beta$ and nourishing the brain environment 
[11-16]. A series of phthalazinone derivatives were synthetized by Irene G. Salado's team, and these compounds are effective to the treatment of Human African trypanosomiasis through the inhibition of PDE4 ${ }^{[17]}$. From the other perspectives, these compounds might be effective for the treatment of AD. What's more, phthalazinone derivatives have been studied as structure correctors to interfere with the structural composition of Apolipoprotein E4 (apo E4) in cultured neurons, which as a result, protect the neurons from Tau hyperphosphorylation, $A \beta$ deposition ${ }^{[18]}$. Besides, the phthalazine derivatives have been applied to neuron cells or animal behavioral experiments to investigate their potential in $A D$ treatment. The results available all shown that the phthalazine had the potency to cure AD by inhibiting AchE, alpha-glycosidase, Cholinesterase (BchE) ${ }^{[19-21]}$. It is of the great potential that phthalazinone derivatives would be effective in the treatment of $A D$, or be lead compounds to develop new drugs.

Network Pharmacology integrates chemistry, pharmacology, pharmacodynamics, and facilitates the mechanistic studies of potential drugs ${ }^{[22]}$. Therefore it is recently popular to be employed to the analysis of potential pharmacological mechanisms of traditional Chinese medicine (TCM) ${ }^{[23,24]}$. Yinchenhao decoction, Potentilla Discolor Bunge, Schisandra Chinensis and many other medicinal herbs have been applied to network pharmacology analysis, resulting in potential pharmacological mechanism of herbs to certain disease ${ }^{[25-27]}$. The core idea of this technology is systematically treating diseases, and this consumption conforms to the actual drug reaction ${ }^{[22]}$. AD, triggered by multi-targets, would be treated more effectively by the multi-targets drug. It is of great significance to apply network pharmacology analysis to compound bioactivity screening.

In this study, the active compounds would be retrieved from literature with certain bioactivity for PDE4. Only those compounds with certain physical properties would be adopted for analysis. The targets prediction,network construction, and gene enrichment analysis would be conducted from the public database. The underlying pharmacological mechanism of phthalazinone derivatives would be helpful to screen the potential bioactivity and extract compounds to develop drugs for AD treatment.

\section{Materials And Methods}

\subsection{Collecting Compounds for Analysis}

The compounds were retrieved from the published literature ${ }^{[17]}$. The compounds' structures were drawn into Pubchem (https://pubchem.ncbi.nlm.nih.gov/) database for search, and their names as well as physical properties were displayed. According to the Lipinski rules, whenever the compounds conform to the criteria: Molecular Weight (MW) less than 500, Hydrogen Bond Donor Count (HB) less than 5 , Hydrogen Bond Acceptor Count (HA) less than 10, the value of Oil-water partition coefficient (cLogP) less than 5 , Rotatable Bond Count (RB) less than 10, they would be more possible to have good absorption and small metabolism after oral administration. Once the compounds' properties disaccord with Lipinski rules for more than two criteria, it is of great possibility that no matter how effective these compounds 
are, they could not be developed into drugs. Those compounds that conformed to the Lipinski rules and could be retrieved from Pubchem database would be employed for this study.

\subsection{Prediction of Compound-Related Targets}

The compound-related targets were predicted depending on chemical similarities and pharmacophore models visa Swiss Target Prediction (http://www.swisstargetprediction.ch/) database ${ }^{[28]}$, Batman TCM (http://bionet.ncpsb.org/batman-tcm/) database ${ }^{[29]}$, and PharmMapper (http://www.lilabecust.cn/pharmmapper/) database ${ }^{[30-32]}$. In the Batman database, those candidate targets with scores given by the target prediction method no less than 20 and $p$ no more than 0.05 were recruited. Targets with Norm Fit more than 0.8000 in PharmMapper database, targets with probability rank top 10 and values greater than zero in Swiss Target Prediction database were also recruited. With the combination of all these targets, about 130 targets were available for later analysis.

\subsection{Identification of AD-Related Targets}

The known AD-related targets were extracted from TTD (Therapeutic Target Database, https://db.idrblab.org/ttd/) database [33], and CTD (Comparative Toxicogenomics Database, http://ctdbase.org/) database ${ }^{[34]}$. Only those targets with inference score no less than 20 in CTD database and clinical trial targets for AD treatment in the TTD database were included. 2630 targets were found to conform to the condition without replicated targets.

\subsection{Scanning of the common targets related to compounds and $A D$ treatment}

All the targets obtained above were standardized as gene symbols and Uniprot IDs by searching from Uniprot (https://www.uniprot.org/) database with "Homo sapiens" species.

Utilizing Venny2.1.0 (http://bioinfogp.cnb.csic.es/tools/venny/) website ${ }^{[35]}$, the common targets both related to compounds and $A D$ treatment were screened.

\subsection{Scanning drugs approved for AD treatment}

Drugbank 5.1.7 (https://www.drugbank.ca/) and TTD database are usually used for the query of drugs and targets. The approved drugs with a clear indication for AD would be adopted, as well as their corresponding targets.

\subsection{Network Construction and Topological Analysis}

The network construction and topological analysis were mainly conducted by STRING 11.0 (https://string-db.org/cgi/input.pl) database and Cytoscape v3.7.2 software. The topological analysis was performed by the Cytoscape online network analyzer module. The parameters of Degree centrality $(\mathrm{DC})$, betweenness centrality (BC), and closeness centrality (CC) would be displayed to evaluate the central properties of the nodes in the network. Those nodes with $D C \geq$ median $D C, B C \geq$ median $B C, C C \geq$ median CC, would be employed as the key targets of compounds. 


\subsection{GO and KEGG Pathway Enrichment analysis}

The GO and KEGG enrichment analysis are of great importance for the study of functional annotation and pathways of selected genes. The GO and KEGG enrichment analysis were carried out by DAVID v6.8 (The Database for Annotation, Visualization and Integrated Discovery, https://david.ncifcrf.gov/) database ${ }^{[36,37]}$, with $p$-value set to less than 0.05 .

\subsection{Study of molecular docking}

The study of molecular docking was performed by iGEMDOCK v2.1. The docking analysis of iGEMDOCK software was based on k-means and hierarchical clustering methods. The shared targets that were related to the same essential pathway or regarded as key targets of compounds would be extracted to molecular docking. As general cases, the default parameters for screening were adopted (population size $=200$, generations $=70$, number of solutions $=2$ ) .

\section{Results}

\subsection{Compounds for Analysis}

In the Pubchem database, six compounds could be retrieved and five of them accord with the Lipinski rules. The compounds' structures and other properties are listed in Table 1. All of the five molecular have molecular weight more than 500 , with other physical properties comply with Lipinski rules. 


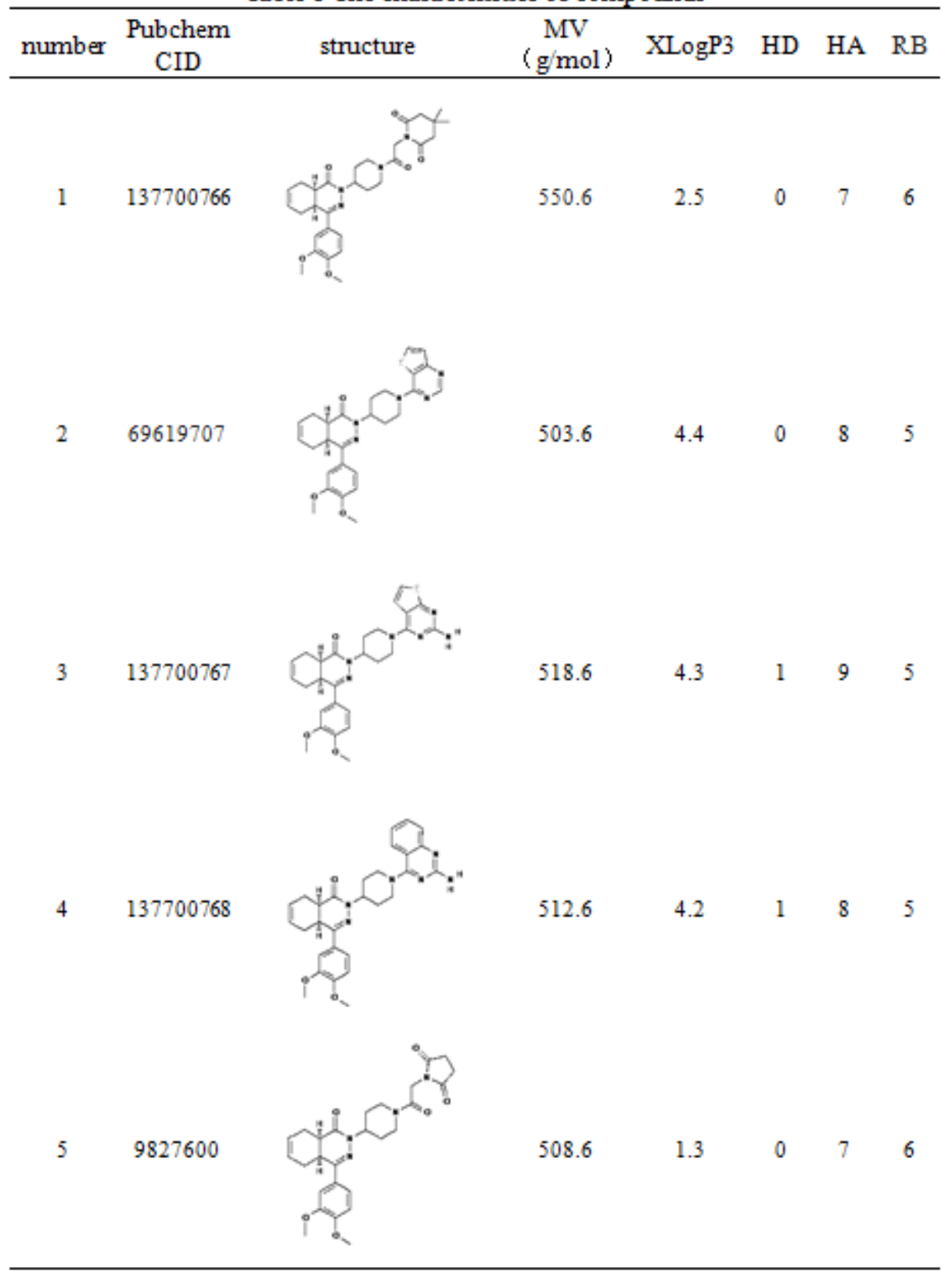

\subsection{Compounds-common targets network of phthalazinone derivatives}

The network of compounds-common targets was constructed by STRING database with minimum required interaction score of 0.400 . The network (Fig. 1) was consisted of 62 nodes (57 common targets and 5 compounds)with a centralization of 0.565 . In this network, it is suggested that all the compounds recruited are possible to be effective for the treatment of $A D$, and compound 2 and compound 3 might enjoy more potential to be drugs for the treatment of $A D$, with a degree of 37,43 respectively. The interactions between phthalazinone derivatives and common targets were as below. And all the compounds employed could interact with either PDE 4B or PDE4D.

\subsection{PPI network of common targets}


To further investigate the interaction between the compound targets, the PPI network was constructed. 57 targets were identified as common targets that both related to AD and phthalazinone derivatives, and 25 targets were regarded to directly interact with common targets. As shown in Fig. 2A, PDE 4D, NOS1, TNF,

INS and other targets in pink were identified as common targets with higher degree value, which to some extent, mean that these targets enjoy more potential to be targets of phthalazinone derivatives to treat AD. According to the topological analysis of the network, INS, TNF, HSP90AA1, NOS1, PTGS2, ADRB2, HSPA1A, CALCA, ARRB1, TRPV1, NFKB1 and F2 were believed to be the key targets among common targets.

The common targets and associated targets were combined and employed to GO and KEGG enrichment analysis. The bubble diagram (Fig. 2B) suggested that the cGMP-PKG signaling pathway (hsa04022), Neuroactive ligand-receptor interaction (hsa04080) and Calcium signaling pathway (hsa04020) were more reliable to be considered as the major pathways that phthalazinone derivatives work on $A D$ treatment. Besides the CAMP signaling pathway (hsa04024) as well as Morphine addition pathway (hsa05032) were also regarded as important pathway for phthalazinone derivatives.

The result of the GO enrichment analysis of combined targets was displayed by a bar chart (Fig. 2C). The red bar represents the biological process (BP), the yellow bar represents cellular component (CC), and the blue bar represents molecular function (MF). As shown in Fig. 2C, it suggested that phthalazinone derivatives took effect by the biological process of signal transduction (GO: 0007165), cell-cell signaling (GO: 0007267) or as cellular component of the plasma membrane (GO: 0005886), cytosol (GO: 0005829), integral component of membrane (GO: 0016021), integral component of plasma membrane (GO: 0005887) $(p<0.01)$. The phthalazinone derivatives could also take part in the molecular function, such as protein binding (GO: 0005515), ATP binding (GO: 0005524), protein homodimerization activity (GO: 0042803) $(p<0.01)$.

\subsection{Drug approved- targets network}

15 kinds of drugs were figured out and approved with a clear indication for AD. The targets concerning were identified at the same time and employed to construct the drug approved-targets network. The network consisted of 72 nodes ( 57 targets and 15 drugs). It is apparent that these targets were prone to enjoy higher interaction (Fig. 3A). According to the topological analysis of this network, GRIN2B, GRIN1, GABRA1, GRIN2A, GABRB2, GLRB, GLRA2 and many other targets had no less than 30 neighbored nodes to interact with. The bubble diagram indicated that Neuroactive ligand-receptor interaction (hsa04080) and Nicotine addiction (hsa05033) pathways are of great essential for the approved drugs to take effect. Besides, Retrograde endocannabinoid signaling (hsa04723), GABAergic synapse (hsa04727) and Morphine addition (hsa05032) pathways were also considered as important in AD treatment (Fig. 3B).

\subsection{Targets-pathways network}


As what has been shown above, the KEGG pathways of phthalazinone derivatives and drugs approved for $A D$ treatment were similar, sharing the same essential pathway, Neuroactive ligand-receptor interaction (hsa04080). To further compare the similarity of targets and pathways between phthalazinone derivatives and approved drugs, the targets-pathways networks were constructed. The pathways employed to analysis were conformed to the condition that $p<0.05$ and associated gene count no less than 3.

Comparing the two figures below, it is notable that the network of approved drugs was more extensively interacted and each pathway enjoyed more gene count. However, the two networks also shared the same pathways and targets. For example, Neuroactive ligand-receptor interaction (hsa04080), Morphine addiction (hsa05032), cAMP signaling pathway (hsa04024), Calcium signaling pathway (hsa04020), Serotonergic synapse (hsa04726), and NF-kappa B signaling pathway (hsa04064) were the same in two networks. Besides, they shared the same targets, including GABRA2, CHRNA7, PTGS1, HTR1A, GABRB3, ADRA1A, NOS1, ADRA2A, PTGS2, SLC6A4 and NFKB1. Among them, the pathway of neuroactive ligandreceptor interaction was considered as essential in both the two networks. PTGS2, NOS1 and NFKB1 targets were also considered as key targets in the PPI network of phthalazinone derivatives; while GABRA2, CHRNA7, HTR1A, GABRB3, ADRA2A targets were found to be associated with neuroactive ligand-receptor interaction pathway. These eight targets would be considered as essential targets in this study.

Table 2

The essential targets

\begin{tabular}{|lll|}
\hline Target & Full name & function \\
\hline NFKB1 & nuclear factor NF-kappa-B p105 subunit & regarded as key targets \\
\hline ADRA2A & Alpha-2A adrenergic receptor & related to essential pathway \\
\hline NOS1 & nitric oxide synthase, brain & regarded as key targets \\
\hline GABRB3 & Gamma-aminobutyric acid receptor subunit beta-3 & related to essential pathway \\
\hline HTR1A & Serotonin 1a (5-HT1a) receptor & related to essential pathway \\
\hline PTGS2 & Prostaglandin G/H synthase 2 & regarded as key targets \\
\hline CHRNA7 & Neuronal acetylcholine receptor subunit alpha-7 & related to essential pathway \\
\hline GABRA2 & Gamma-aminobutyric acid receptor subunit alpha-2 & related to essential pathway \\
\hline
\end{tabular}

\subsection{Molecular docking study}

The molecular docking result was calculated on the generic evolutionary method (GA) and presented by binding energy. Those with lower binding energy enjoy higher stability. The result indicated that phthalazinone derivatives could all interacted with these essential targets and keep more stable than associated bounded ligands (Fig. 5). NOS1 (4UCH) could be interacted and keep stable by compound 1, 
compound 3 and compound 4 . In contrast with other phthalazinone derivatives, compound 1 and compound 4 enjoy higher binding capacity with the essential targets.

\section{Discussion}

\subsection{Phthalazinone derivatives' potential for AD treatment}

$A D$, triggered by multi-targets, is prevalent among the aged and is of great necessity to develop drugs for treatment. Several traditional herbs and associated prescriptions have been employed to explore their potential mechanisms to treat AD by network pharmacology analysis and molecular docking study $[38,39]$. In this study, the in silico analysis all suggested that the phthalazinone derivatives employed were potential to be lead compounds to develop drugs for AD treatment. This finding conformed to the conclusions in published literatures. According to the available literatures, compounds with phthalazinone or phthalazine original nucleus were effective to treat $A D$, no matter on neuron cell groups or animal models ${ }^{[40]}$. For example, phthalazine derivates such as Hydralazine and $\triangle 5 \mathrm{IA}$, was found to be available to reduce oxidative damage and $A \beta$ misfolding or function as an inverse agonist of 5 subunitcontaining Gamma-aminobutyric acid receptors (a5GABAARs) to retard the aggression of $A \beta^{[41-43]}$. What's more, Zopolestat, a phthalazinone derivative, had been reported to be available to inhibit $A \beta$ induced neuroinflammation by regulating NF-KB and other signaling pathways, enjoying great potency to be drugs for AD treatment ${ }^{[44]}$.

\subsection{Potential mechanism of phthalazinone derivatives}

The gene enrichment analysis revealed that the candidate phthalazinone derivatives could interact with PDE4 and other targets, initiating cAMP signaling pathway, neuroactive ligand-receptor interaction, morphine addiction, and calcium signaling pathway (Fig. 4A, Fig. 2B), to take into effect in AD treatment. Such potential pathways not only were considered as reliable to explain the mechanisms of approved $A D$ drugs (Fig. 3B, $\mathrm{p}<0.01$ ), but also could be extracted from the published literatures.

Neuroactive ligand-receptor interaction pathway could help to increase the release of dopamine, resulting in the protection of brain nerve ${ }^{[45]}$. Recent studies have reported that the opioid system was relevant to the development of various neurodegenerative diseases. The opioid drugs including morphine could induce intestinal dysbiosis and trigger changes in the central nervous system, interrupting the development of $A D{ }^{[46]}$. As what has been known, PDE 4 subtype is the cAMP-specific phosphodiesterase and plays an essential role in the regulation of intracellular levels of cAMP. It is of great benefit to nourish the brain neuron and inhibit the aggression of $A \beta$ by interrupting cAMP. By triggering cAMP/Protein Kinase A (PKA)/ cAMP-Response Element-Binding Protein (CREB)/ Brain-Derived Neurotrophic Factor

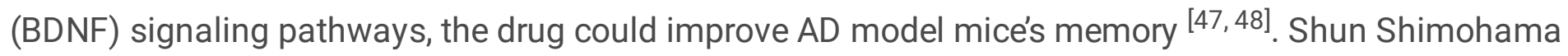
and Jun Kawamata indicated that the influx of $\mathrm{Ca}^{2+}$ would contribute to the intracellular signaling transduction and inhibit the aggression of $A \beta^{[49]}$. That also means,calcium signaling pathway would influence the development of AD. A literature review had already summarized that calcium channel could 
relate to all $A D$ pathologies and calcium signaling pathway is a promising breakthrough for $A D$ treatment [50].

The GO and KEGG enrichment analysis (Fig. 2) suggested that the phthalazinone derivatives mainly took into effect by improving energy imbalance in the brains of $A D$ patients.

\subsection{Molecular docking analysis}

There were two patterns for the preparation of protein (based on the current file and based on the bounded site) in molecular docking. The binding energy in these two patterns was nearly the same (STable 1). There was no bounded ligand in the target NFKB1 (2061), and the pattern that preparing protein based on the current file was adopted to keep the consistency in this study. The binding energy among these phthalazinone derivatives were of no significant difference (Fig. 5). It is possible that the phthalazinone core structure with 2'-pyridine, and 8'--methoxyphenyl ensured these phthalazinone derivatives to interact with essential targets stably. The substituent group on pyridine made a little difference in the interaction. For example, hexatomic ring substituent enjoys better interaction stability than the furan-one. If there is an electrophilic group on the substituent and could interact with the receptor to form a hydrogen bond, this phthalazinone derivative would be easier to interact with.

\subsection{Significance and application}

Network pharmacology analysis was first developed by Hopink in 2007, and today is popular in the study of the potential pharmacological mechanism of traditional Chinese medicine ${ }^{[51]}$. According to the methodology and theory of network pharmacology analysis, it could be adopted to initially screen the bioactivity of compounds and investigate the potential mechanism ${ }^{[22,52]}$. Different from other literatures about phthalazinones, this study employed network pharmacology analysis and molecular docking to suggest that those compounds could be effective in AD treatment. And network pharmacology analysis of the approved drugs was employed to further demonstrate the reliability of such bioactivity and mechanism. By network pharmacology analysis and molecular docking study, not only the potential targets and pathways, but also the binding capacity and structures-activity relationships were figured out.

The analytical pattern could be available to screen specific bioactivity of the component group of traditional Chinese medicine and figure out the underlying pharmacological mechanism. It is also of great benefit to employing this pattern to discover new indications for the approved drug.

Network pharmacology analysis is a methodology based on the statistic posed on databases or published literatures, it is not a perfect match with the actual situation. And the targets we found on the database were displayed without actual interaction (agonist or antagonist) ${ }^{[53]}$. Pharmacodynamics research on $A D$ animal models still needs to be conducted to verify the bioactivity of phthalazinone derivatives.

\section{Conclusion}


In this study, the analysis of phthalazinone derivatives suggested that these candidates were prone to enjoy bioactivity to treat $A D$ and keep each interaction stably. The network pharmacology analysis of the approved drugs further testified the reliability of phthalazinone derivatives as drugs for AD treatment. All the analysis indicated that these phthalazinone derivatives were potential to be lead compounds to develop drugs for $A D$ treatment. The $G O$ enrichment analysis showed that the common targets could function involve in various biological processes, cellular components and molecular functions. The GO and KEGG enrichment analysis of common targets and associated targets suggested that these phthalazinone derivatives were effective mainly by interacting with signal transduction process, ATP binding, neuroactive ligand-receptor interaction, morphine addiction, cAMP signaling pathway, and calcium signaling pathway. The interactions above are conformed to the published literatures and could be employed to the treatment for AD. Network pharmacology analysis is usually applied to the potential pharmacological mechanism of traditional Chinese medicine. We think it is a good attempt to connect network pharmacology analysis and molecular docking with bioactivity screening. It is of great benefit to broaden the scale of network pharmacology applications.

\section{Declarations}

\section{Ethics approval and consent to participate}

Not applicable. This manuscript does not report on or involve the use of any animal or human data or tissue.

\section{Consent for publication}

Not applicable. This manuscript does not contain data from any individual person.

\section{Availability of data and materials}

All data are available in the manuscript and are shown in tables, figures, and supplemental files.

\section{Conflict of interest}

There is no conflict of interest in this work.

\section{Funding}

Not applicable. There was no funding supported for this research.

\section{Acknowledgements}

I thank my tutor for manuscript writing support and appreciate for the guidance offered by Dr. Xie Yuanyuan to revise this paper. Besides, I quite appreciate for my family's support and patience over my career. 


\section{References}

1. Association Alzheimer's. 2016 Alzheimer's disease facts and figures[J]. Alzheimers Dement, 2016,12(4):459-509.doi: 10.1016/j.jalz.2016.03.001.

2. Kit Media. Primary Care Physicians on the Front Lines of Diagnosing and Providing Alzheimer's and Dementia Care: Half Say Medical Profession Not Prepared to Meet Expected Increase in Demands[EB/OL]. https://www.alz.org/news/2020/primary-care-physicians-on-the-front-lines-ofdiag?_ga=2.191767662.538060142.1591935791-1222386042.1591935791.

3. Pan T, Xie S, Zhou Y, et al. Dual functional cholinesterase and PDE4D inhibitors for the treatment of Alzheimer's disease: Design, synthesis and evaluation of tacrine-pyrazolo[3,4-b]pyridine hybrids[J]. Bioorg Med Chem Lett. 2019;29(16):2150-2. doi:10.1016/j.bmcl.2019.06.056.

4. Hu J, Pan T, An B, et al. Synthesis and evaluation of clioquinol-rolipram/roflumilast hybrids as multitarget-directed ligands for the treatment of Alzheimer's disease[J]. Eur J Med Chem. 2019;163:512-26. doi:10.1016/j.ejmech.2018.12.013.

5. Cui SY, Yang MX, Zhang YH, et al. Protection from Amyloid beta Peptide-Induced Memory, Biochemical, and Morphological Deficits by a Phosphodiesterase-4D Allosteric Inhibitor[J]. J Pharmacol Exp Ther. 2019;371(2):250-9. doi:10.1124/jpet.119.259986.

6. Hogh P [Alzheimer's disease][J]. Ugeskr Laeger, 2017,179(12).pmid: 28330540.

7. Silva MVF, Loures CMG, Alves LCV, et al. Alzheimer's disease: risk factors and potentially protective measures[J]. J Biomed Sci. 2019;26(1):33. doi:10.1186/s12929-019-0524-y.

8. Kumar A, Singh A. Ekavali. A review on Alzheimer's disease pathophysiology and its management: an update[J]. Pharmacol Rep. 2015;67(2):195-203. doi:10.1016/j.pharep.2014.09.004.

9. Zhang N, Gordon ML. Clinical efficacy and safety of donepezil in the treatment of Alzheimer's disease in Chinese patients[J]. Clin Interv Aging. 2018;13:1963-70. doi:10.2147/CIA.S159920.

10. Sinayiyao. In recent years, clinical trials of new $A D$ drugs have ended in failure. What else can biomedicine do in the future?[EB/OL]. https://chuansongme.com/n/2377862352330.

11. Wu Y, Li Z, Huang YY, et al. Novel Phosphodiesterase Inhibitors for Cognitive Improvement in Alzheimer's Disease[J]. J Med Chem. 2018;61(13):5467-83. doi:10.1021/acs.jmedchem.7b01370.

12. Ricciarelli R, Brullo $C$, Prickaerts J, et al. Memory-enhancing effects of GEBR-32a, a new PDE4D inhibitor holding promise for the treatment of Alzheimer's disease[J]. Sci Rep. 2017;7:46320. doi:10.1038/srep46320.

13. Zhang MZ, Zhou ZZ, Yuan X, et al. Chlorbipram: a novel PDE4 inhibitor with improved safety as a potential antidepressant and cognitive enhancer[J]. Eur J Pharmacol. 2013;721(1-3):56-63. doi:10.1016/j.ejphar.2013.09.055.

14. Zhang $\mathrm{C}$, Cheng $\mathrm{Y}$, Wang $\mathrm{H}$, et al. RNA interference-mediated knockdown of long-form phosphodiesterase-4D (PDE4D) enzyme reverses amyloid-beta42-induced memory deficits in mice[J]. J Alzheimers Dis. 2014;38(2):269-80. doi:10.3233/JAD-122236. 
15. Tibbo AJ, Tejeda GS, Baillie GS. Understanding PDE4's function in Alzheimer's disease; a target for novel therapeutic approaches[J]. Biochem Soc Trans. 2019;47(5):1557-65. doi:10.1042/BST20190763.

16. Prickaerts J, Heckman PRA, Blokland A. Investigational phosphodiesterase inhibitors in phase I and phase II clinical trials for Alzheimer's disease[J]. Expert Opin Investig Drugs. 2017;26(9):1033-48. doi:10.1080/13543784.2017.1364360.

17. Salado IG, Singh AK, Moreno-Cinos C, et al. Lead Optimization of Phthalazinone Phosphodiesterase Inhibitors as Novel Antitrypanosomal Compounds[J]. J Med Chem. 2020;63(7):3485-507. doi:10.1021/acs.jmedchem.9b00985.

18. Chen HK, Liu Z, Meyer-Franke A, et al. Small molecule structure correctors abolish detrimental effects of apolipoprotein E4 in cultured neurons[J]. J Biol Chem. 2012;287(8):5253-66. doi:10.1074/jbc.M111.276162.

19. Gao CZ, Dong W, Cui ZW, et al. Synthesis, preliminarily biological evaluation and molecular docking study of new Olaparib analogues as multifunctional PARP-1 and cholinesterase inhibitors[J]. J Enzyme Inhib Med Chem. 2019;34(1):150-62. doi:10.1080/14756366.2018.1530224.

20. Taslimi P, Turhan K, Turkan F, et al. Cholinesterases, alpha-glycosidase, and carbonic anhydrase inhibition properties of $1 \mathrm{H}$-pyrazolo[1,2-b]phthalazine-5,10-dione derivatives: Synthetic analogues for the treatment of Alzheimer's disease and diabetes mellitus[J]. Bioorg Chem. 2020;97:103647.doi. 10.1016/j.bioorg.2020.103647.

21. Jalili-Baleh L, Nadri $H$, Moradi A, et al. New racemic annulated pyrazolo[1,2-b]phthalazines as tacrinelike AChE inhibitors with potential use in Alzheimer's disease[J]. Eur J Med Chem. 2017;139:280-9. doi:10.1016/j.ejmech.2017.07.072.

22. Li S, Zhang B. Traditional Chinese medicine network pharmacology: theory, methodology and application[J]. Chin J Nat Med. 2013;11(2):110-20. doi:10.1016/S1875-5364(13)60037-0.

23. Zhao Fangqing Zhai Fei Xiang Rongwu. Visualized analysis of the research trend of network pharmacology[J]. China Journal of Traditional Chinese Medicine Pharmacy. 2018;33(07):3099-103.

24. Liu Yanfei Sun Mingyue Zhao Yingke. Network pharmacology in drug repositioning of Chinese medicines: application status and thinking[J]. Chinese Journal of Evidence-Based Medicine., 2017(17(11)):1344-1349.doi:10.7507/1672-2531.201707013.

25. Huang J, Cheung $F$, Tan HY, et al. Identification of the active compounds and significant pathways of yinchenhao decoction based on network pharmacology[J]. Mol Med Rep. 2017;16(4):4583-92. doi:10.3892/mmr.2017.7149.

26. Wang N, Zhu F, Shen M, et al. Network pharmacology-based analysis on bioactive anti-diabetic compounds in Potentilla discolor bunge[J]. J Ethnopharmacol. 2019;241:111905.doi. 10.1016/j.jep.2019.111905.

27. Cheng Kun Sun Feiyi Dong Jian. Network Pharmacology-Based Study on the Mechanism of Schisandra chinensis for Treating Alzheimer's Disease[J]. Indian J Pharmacol. 2020;52(2):94-101. doi:10.4103/ijp.IJP_515_19. 
28. Antoine Daina Olivier Michielin Vincent Zoete. SwissTargetPrediction: updated data and new features for efficient prediction of protein targets of small molecules[J]. Nucleic Acids Res, 2019(47):W357-64.doi:10.1093/nar/gkz382.

29. Liu Z, Guo F, Wang Y. BATMAN-TCM: a Bioinformatics Analysis Tool for Molecular mechANism of Traditional Chinese Medicine. [J] Sci Rep, 2016(6:21146.).doi:10.1038/srep21146.

30. Xiaofeng Liu Sisheng Ouyang Biao Yu. PharmMapper Server: a web server for potential drug target identification using pharmacophore mapping approach[J]. Nucleic Acids Res, 2010(38):W609-14. doi:10.1093/nar/gkq300.

31. Xia Wang Chenxu Pan Jiayu Gong., Li H. Enhancing the Enrichment of Pharmacophore-Based Target Prediction for the Polypharmacological Profiles of Drugs. [J]. J Chem Inf Model, 2016(56):117583.doi:10.1021/acs.jcim.5b00690.

32. Xia Wang Yihang Shen Shiwei Wang. PharmMapper 2017 update: a web server for potential drug target identification with a comprehensive target pharmacophore database. [J]. Nucleic Acids Res, 2017(45):W356-60.doi:10.1093/nar/gkx374.

33. Yunxia Wang Song Zhang Fengcheng Li. Therapeutic Target Database 2020: Enriched Resource for Facilitating Research and Early Development of Targeted Therapeutics[J]. Nucleic Acids Res, 2020(48(D1)):D1031-41.doi:10.1093/nar/gkz981.

34. Allan Peter Davis Cynthia J. Grondin Robin. The Comparative Toxicogenomics Database: Update 2019[J]. Nucleic Acids Res, (47(D1)):D948-D954.doi: 10.1093/nar/gky868.

35. Oliveros JC. An interactive tool for comparing lists with Venn's diagrams[EB/OL]. https://bioinfogp.cnb.csic.es/tools/venny/index.html.

36. Da Wei Huang Brad T. Sherman Richard. Systematic and Integrative Analysis of Large Gene Lists Using DAVID Bioinformatics Resources[J]. Nat Protoc, 2009(4(1)):4457.doi:10.1038/nprot.2008.211.

37. Da Wei Huang Brad T. Sherman Richard. Bioinformatics Enrichment Tools: Paths Toward the Comprehensive Functional Analysis of Large Gene Lists[J]. 2009(37(1)):1-13.doi:

10.1093/nar/gkn923.

38. Xiao H, Qin X, Wan J, et al. Pharmacological Targets and the Biological Mechanisms of Formononetin for Alzheimer's Disease: A Network Analysis[J]. Med Sci Monit. 2019;25:4273-7. doi:10.12659/MSM.916662.

39. Pang XC, Kang, Fang JS, et al. Network pharmacology-based analysis of Chinese herbal Naodesheng formula for application to Alzheimer's disease[J]. Chin J Nat Med. 2018;16(1):53-62. doi:10.1016/S1875-5364(18)30029-3.

40. Shen L, Yan M, He L. D5 receptor agonist 027075 promotes cognitive function recovery and neurogenesis in a Abeta1-42-induced mouse model[J]. Neuropharmacology. 2016;105:72-83. doi:10.1016/j.neuropharm.2016.01.008.

41. Vinnakota C, Govindpani K, Tate WP, et al. An 5 GABAA Receptor Inverse Agonist, 5IA, Attenuates Amyloid Beta-Induced Neuronal Death in Mouse Hippocampal Cultures[J]. Int J Mol Sci, 
2020,21(9).doi:10.3390/ijms21093284.

42. Maheshwari M, Roberts JK, Desutter B, et al. Hydralazine modifies Abeta fibril formation and prevents modification by lipids in vitro[J]. Biochemistry. 2010;49(49):10371-80. doi:10.1021/bi101249p.

43. Burcham PC, Kaminskas LM, Tan D, et al. Carbonyl-scavenging drugs \& protection against carbonyl stress-associated cell injury[J]. Mini Rev Med Chem. 2008;8(4):319-30. doi:10.2174/138955708783955953.

44. Song XM, Yu Q, Dong X, et al. Aldose reductase inhibitors attenuate beta-amyloid-induced TNF-alpha production in microlgia via ROS-PKC-mediated NF-kappaB and MAPK pathways[J]. Int Immunopharmacol. 2017;50:30-7. doi:10.1016/j.intimp.2017.06.005.

45. Su SY, Hsieh CL, Wu SL, et al. Transcriptomic analysis of EGb 761-regulated neuroactive receptor pathway in vivo[J]. J Ethnopharmacol. 2009;123(1):68-73. doi:10.1016/j.jep.2009.02.027.

46. Rueda-Ruzafa L, Cruz F, Cardona D, et al. Opioid system influences gut-brain axis: Dysbiosis and related alterations[J]. Pharmacol Res. 2020;159:104928.doi. 10.1016/j.phrs.2020.104928.

47. Amidfar M, de Oliveira J, Kucharska E, et al. The role of CREB and BDNF in neurobiology and treatment of Alzheimer's disease[J]. Life Sci. 2020;257:118020. doi:10.1016/j.Ifs.2020.118020.

48. Kim SK, Ko YH, Lee SY, et al. Memory-enhancing effects of 7,3',4'-trihydroxyisoflavone by regulation of cholinergic function and BDNF signaling pathway in mice[J]. Food Chem Toxicol. 2020;137:111160.doi. 10.1016/j.fct.2020.111160.

49. Shimohama S, Kawamata J. Roles of Nicotinic Acetylcholine Receptors in the Pathology and Treatment of Alzheimer's and Parkinson's Diseases[J]. 2018:137-158.doi: 10.1007/978-981-108488-1_8.

50. Tong BC, Wu AJ, Li M, et al. Calcium signaling in Alzheimer's disease \& therapies[J]. Biochim Biophys Acta Mol Cell Res. 2018;1865(11 Pt B):1745-60. doi:10.1016/j.bbamcr.2018.07.018.

51. Hopkins AL. Network pharmacology[J]. Nat Biotechnol. 2007;25(10):1110-1. doi:10.1038/nbt10071110.

52. Hopkins AL. Network pharmacology: the next paradigm in drug discovery[J]. Nat Chem Biol. 2008;4(11):682-90. doi:10.1038/nchembio.118.

53. Guoan Luo Y, Wang X. Fan, et al. Research Strategy and Practice from Clinical Reality, Targeting at Signaling Pathways for the Innovative Compound Drug--The Sixth Discussion on the Proposal of Holistic Systems Medicine[J]. Modernization of Traditional Chinese Medicine and Materia MedicaWorld Science Technology. 2018;20(07):1047-68. doi:10.11842/wst.2018.07.001.

\section{Figures}




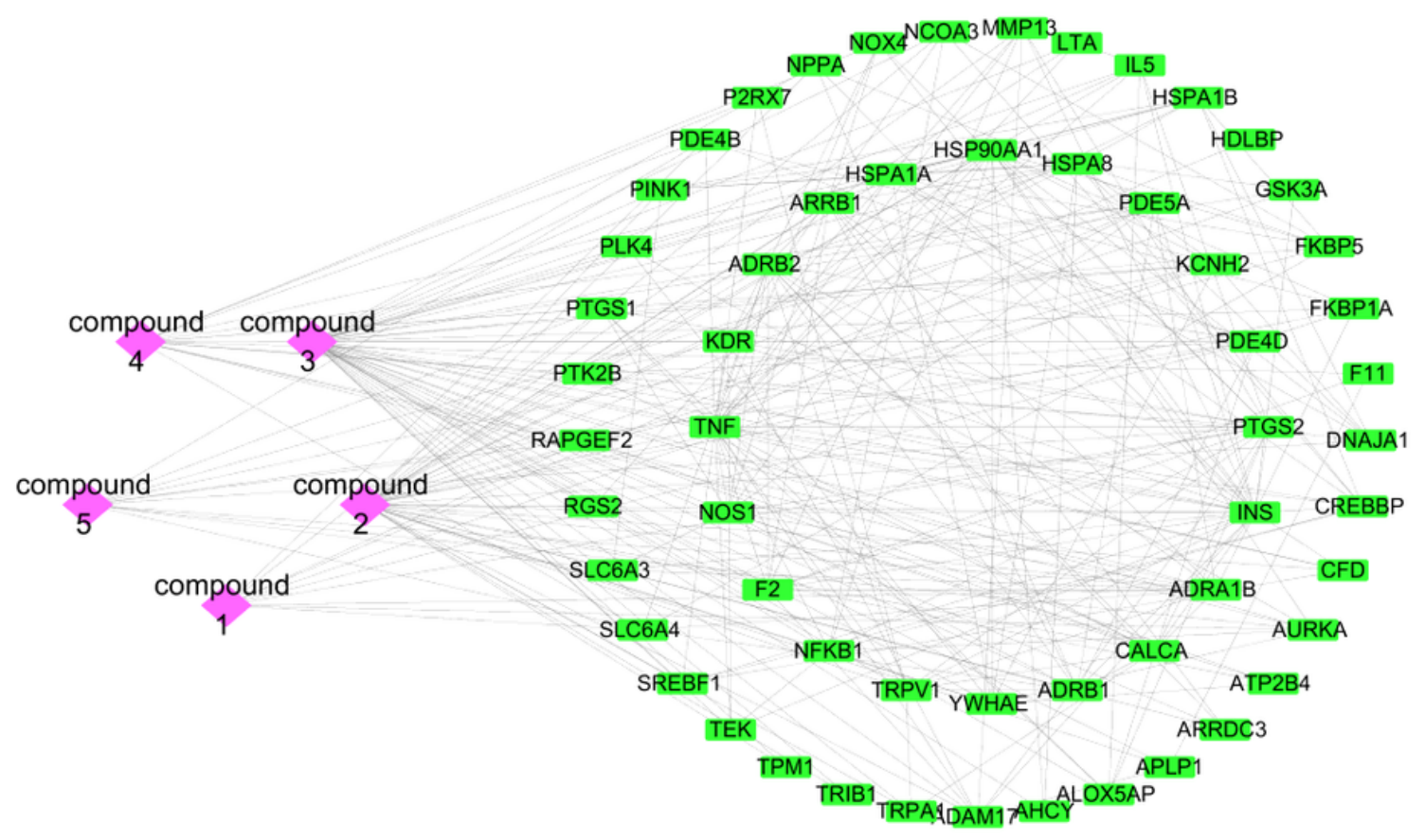

\section{Figure 1}

Compounds-common targets network: The purple Diamond represents the phthalazinone derivatives; the green Round Rectangle represents the common targets. The nodes in inner circle enjoy higher degree. 

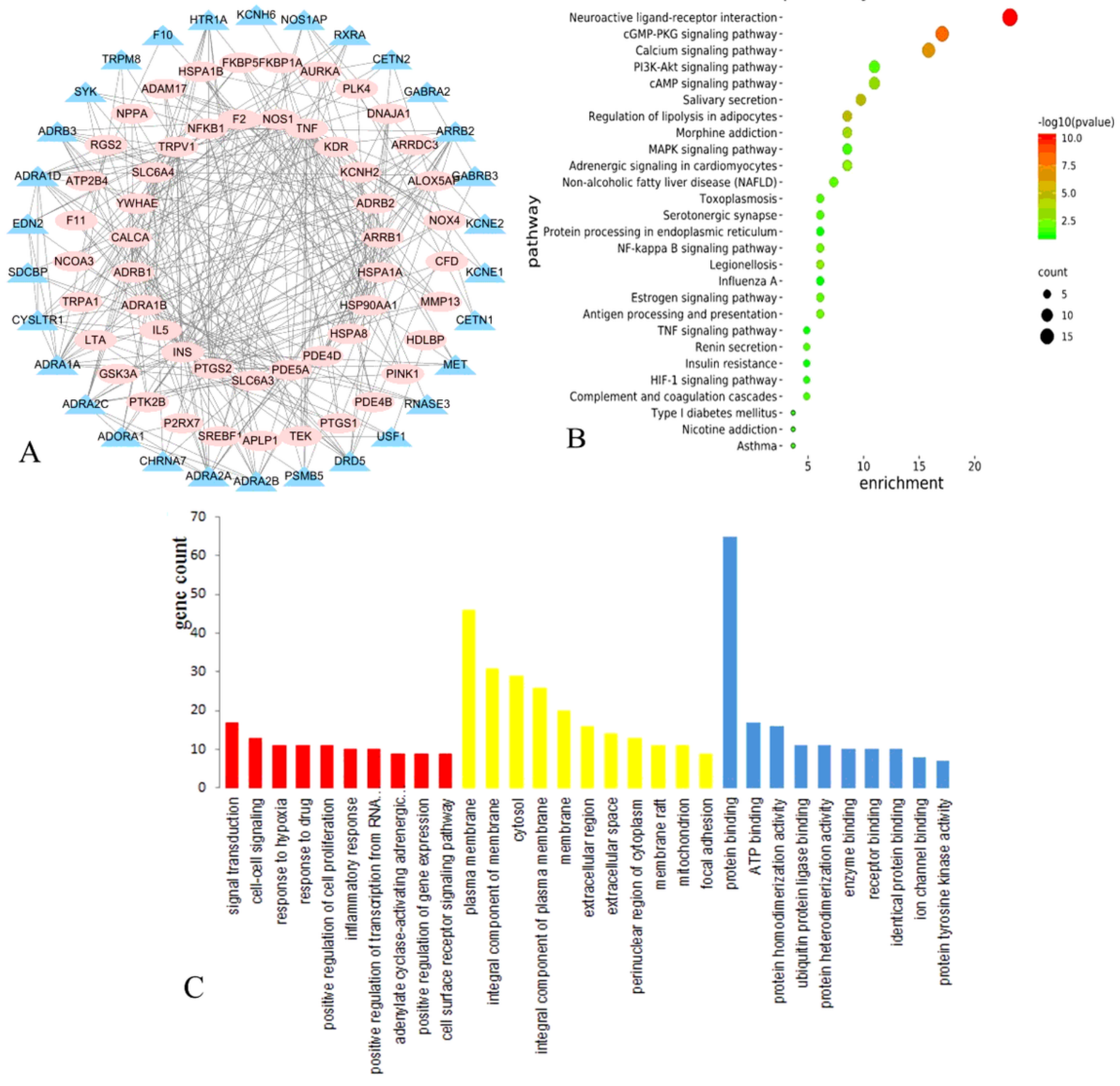

$\mathrm{BP}$

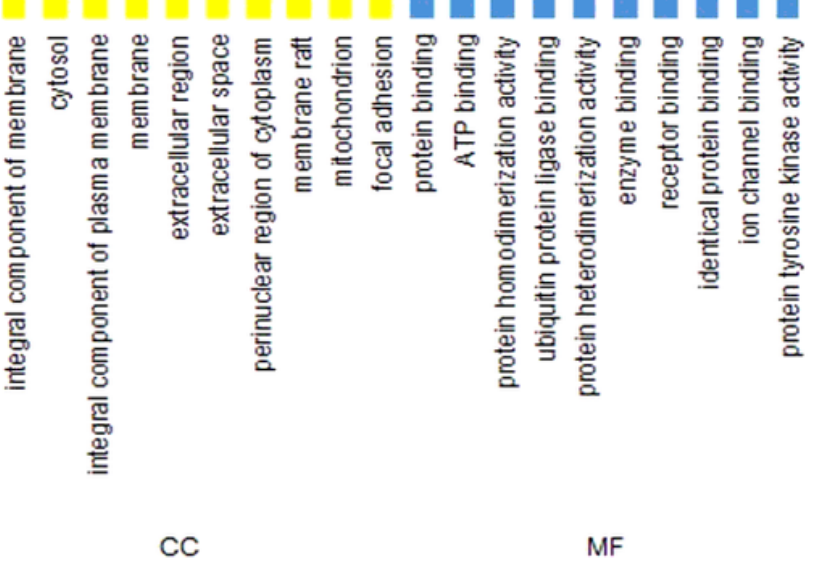

Figure 2

PPI network, GO, and KEGG enrichment analysis. (A) PPI network. The pink ellipse represents common targets, and the targets in the inner circle enjoyed high degree value; the blue triangle represents associated targets. (B) Bubble diagram of KEGG pathways. The $x$-axis represents the gene count ratio, while the $y$-axis represents pathway terms. The bubble's color represents the significance level $(p)$ of corresponding pathways: the significance level decreases ( $p$-value increases) from red to green. And the bubble's size represents the gene count of the pathway. (C) GO enrichment analysis. The red bars 
represent the analysis of $\mathrm{BP}$, the yellow ones represent the analysis of $\mathrm{CC}$, and the blue ones represent the analysis of MF.
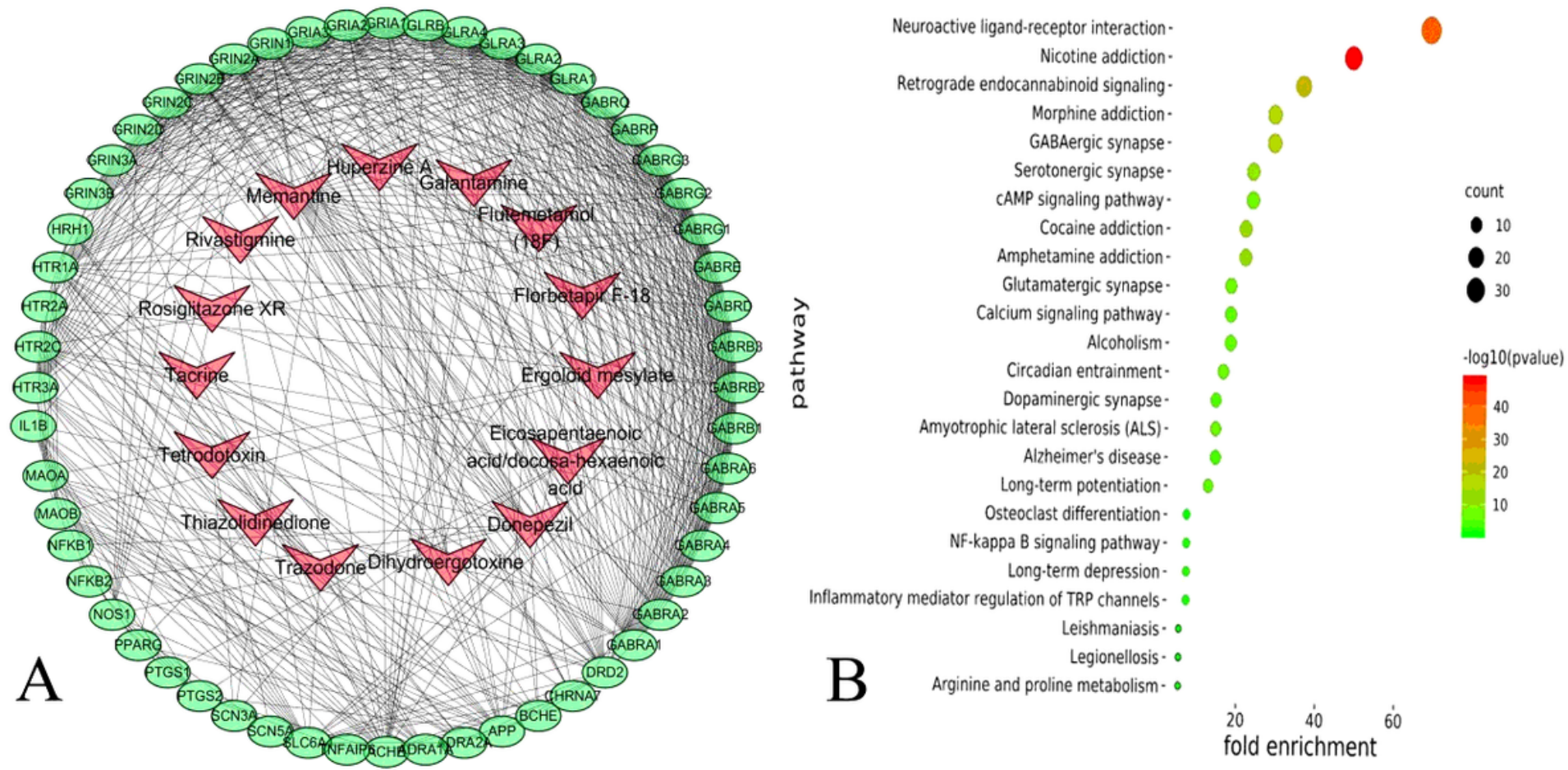

\section{Figure 3}

Drug approved-targets network and KEGG enrichment analysis. (A) The drug approved--targets network. The red $\mathrm{V}$ represents drugs approved for $A D$ treatment; the green ellipse represents drug-related targets. (B) Bubble diagram of KEGG pathways. The x-axis represents the gene count ratio, while the y-axis represents pathway terms. The bubble's color represents the significance level (p) of corresponding pathways: the significance level decreases ( $p$-value increases) from red to green. And the bubble's size represents the gene count of each pathway.
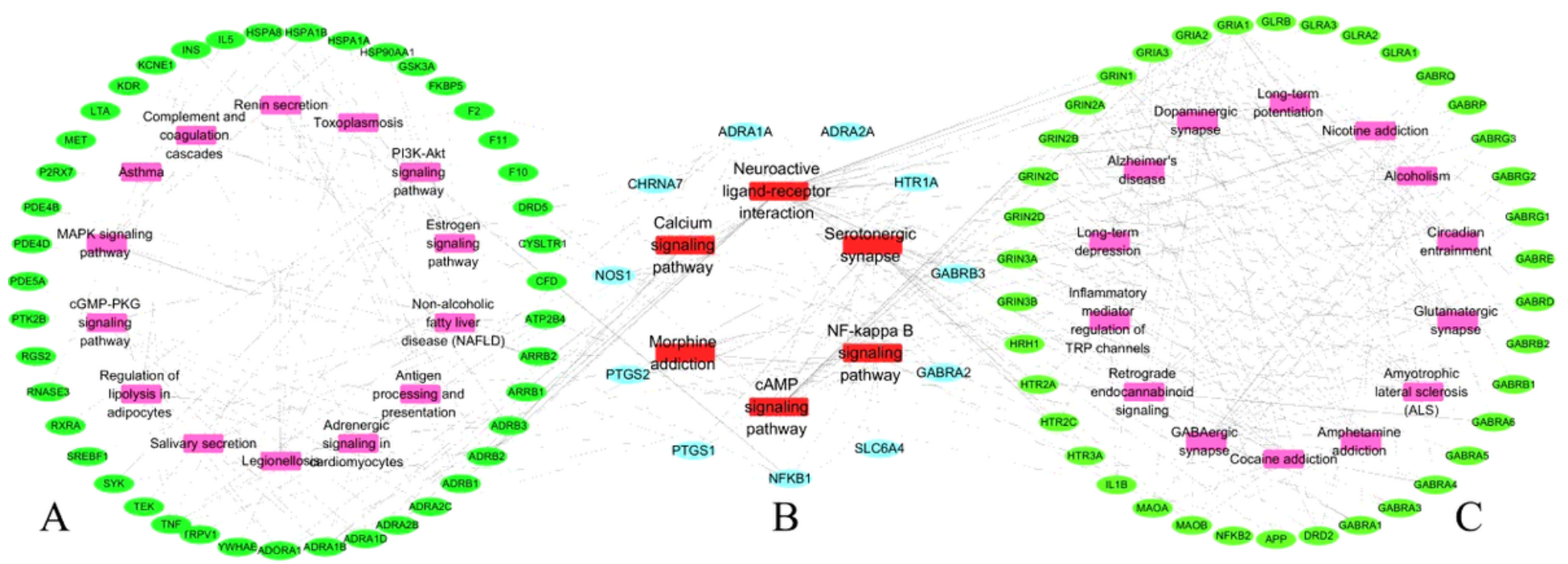

Figure 4 


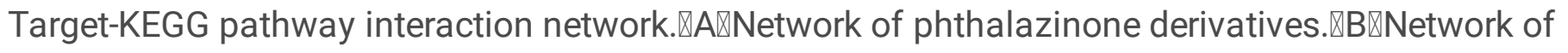
common targets and common pathways. (C) Network of approved drugs. The nodes in rectangle represent pathways while the nodes in ellipse represent targets. The shared targets were in dark blue and the shared pathways were in red in the inner circle.

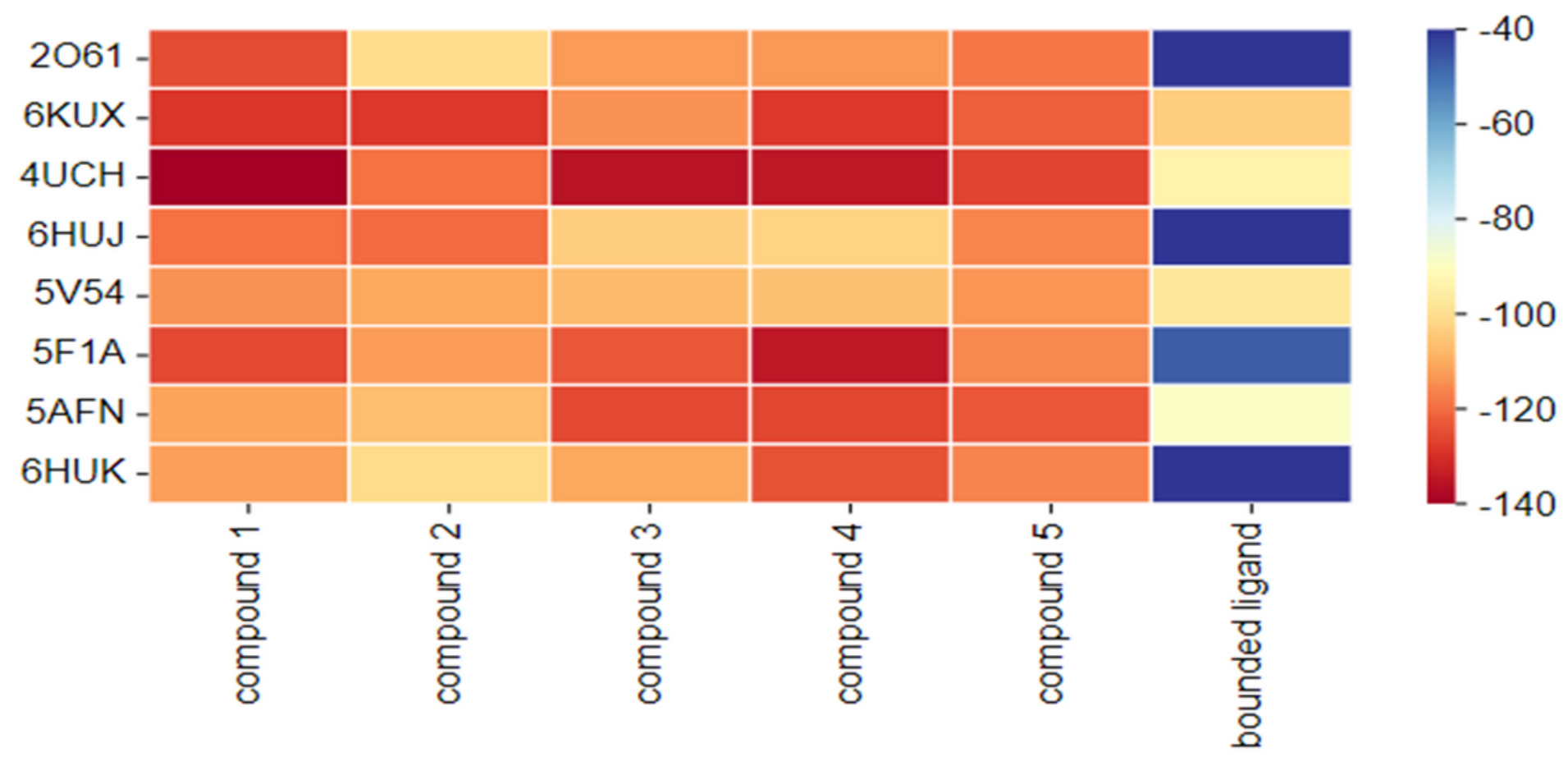

Figure 5

Heat map of binding energy between phthalazinone derivatives and the essential targets in molecular docking study. The heat map was depicted based on binding energy.

\section{Supplementary Files}

This is a list of supplementary files associated with this preprint. Click to download.

- renamedb537e.xlsx

- renamed03f48.xlsx 